\title{
Automatic tuning of data placement and load balancing in disk arrays
}

\section{Report}

\section{Author(s):}

Zabback, Peter; Weikum, Gerhard; Scheuermann, Peter I.

Publication date:

1992

\section{Permanent link:}

https://doi.org/10.3929/ethz-a-000636494

\section{Rights / license:}

In Copyright - Non-Commercial Use Permitted

\section{Originally published in:}

ETH, Eidgenössische Technische Hochschule Zürich, Departement Informatik, Institut für Informationssysteme 175175 


\section{EII}

Eidgenössische

Technische Hochschule

Zürich
Departement Informatik Institut für Informationssysteme
Peter Scheuermann Gerhard Weikum Peter Zabback
Automatic Tuning of Data

Placement and Load Balancing in Disk Arrays

April 1992 
Authors' addresses:

Institut für Informationssysteme

ETH Zentrum, CH-8092 Zurich, Switzerland

(C) 1992 Departement Informatik, ETH Zürich 


\title{
Automatic Tuning of Data Placement and Load Balancing in Disk Arrays*
}

\author{
Peter Scheuermann** \\ Department of Elec. Eng. and Computer Science \\ Northwestern University \\ Evanston, IL 60208 \\ Gerhard Weikum and Peter Zabback \\ Information Systems - Databases \\ Department of Computer Science \\ ETH Zurich \\ CH-8092. Zurich, Switzerland
}

\begin{abstract}
Large arrays of small disks are providing an attractive approach for high performance I/O -systems. They allow for low-cost, reliable storage and can achieve higher throughput compared to large disks. However, in order to make effective use of the commercially available architectures, it is necessary to develop intelligent software tools that allow automatic tuning of the disk arrays to varying workloads. In this paper we describe an integrated set of algorithms and the implementation of a file manager for automatic file partitioning and - allocation and for load balancing on disk arrays. Our approach consists of modular building blocks that can be invoked independently of each other; thus, algorithms for file allocation and disk load balancing can be used regardless of whether striping is employed or not. Our heuristic method for file partitioning aims to determine the optimal striping unit of a file, depending on the access characteristics of the file and the overall workload. Our heuristic method for file allocation and load balancing deals with dynamically changing access frequencies of files by reallocating file extents, thus "cooling down" hot disks. In addition, the method takes into account the fact that some files exhibit periodical access patterns, and considers explicitly the cost of performing the cooling operations.
\end{abstract}

*This report will appear in: Database Systems for Next-Generation Applications - Principles and Practice, Advanced Database Research and Development Series (edited by W. Kim and Y. Kambayashi), World Scientific Publications.

*"This work was performed while the author was visiting with ETH Zurich. 


\section{Introduction}

Large arrays of small disks (also known as RAIDs) provide an attractive alternative over large disks in terms of cost, volume and power $[8,12,19]$. In addition, disk arrays provide the potential for high performance I/O by exploiting the bandwidth of several disks to service a single logical request or multiple independent requests in parallel. High data reliability can be achieved through techniques that introduce data redundancy by using error-correcting codes or variations of mirroring $[7,10,16]$. Disk array technology is rapidly evolving in the commercial market as indicated by a number of products and product plans of startup companies (e.g., Maximum Strategy, Array Technology), as well as those of major computer vendors $[11,15]$.

\subsection{Disk Array Architectures}

In principle, we can distinguish three types of disk array architectures [11,12]. In a synchronous disk array [21], all disks are synchronized with respect to spindle rotation and arm movement. Thus, the disk array appears as a single logical disk with increased bandwidth (i.e., data transfer rate), since all disks can transfer data in parallel. The increased hardware complexity of this architecture pays off only for requests that are large enough to benefit from the increased transfer rate. Thus, typical applications for synchronous disk arrays are in the supercomputing area.

An asynchronous disk array is very similar to a synchronized one, except that there is no hardware support for spindle synchronization. Here again the disk array is used as a single logical disk, and all disks are involved in servicing an $\mathrm{I} / \mathrm{O}$ request.

In a loosely coupled disk array, each disk can be accessed individually. by the operating system. Thus, a loosely coupled disk array can service many independent $I / O$ requests in parallel, in order to improve the throughput of the $1 / O$ requests; alternatively a subset of the disks can service a single request in parallel, in order to decrease the transfer time of a request. This architecture is similar to conventional multi-disk systems (also known as disk farms). However, loosely coupled disk arrays are based on inexpensive small disks and standardized channel architectures (e.g., SCSI-2, HIPPI) rather than high-end technology and proprietary interconnections. 
In our work, we assume that the operating system can access disks individually; hence our algorithms for automatic tuning of data placement are applicable to both disk arrays with loosely coupled disks and to disk farms.

\subsection{I/O Parallelism}

In order to effectively exploit the potential for I/O parallelism in a disk array, files must be partitioned and distributed across disks. File striping or declustering $[13,14,19,20]$ is a technique for file organization which divides a file into runs of logically consecutive blocks (called "stripes") which are then spread across a number of disks in order to reduce the transfer time of a single request or to improve the throughput for multiple requests. The striping unit denotes the number of logically consecutive data blocks stored per disk, and the degree of declustering of a file denotes the number of disks over which a file is spread.

The degree to which the potential I/O parallelism of a disk array can be effectively exploited in an application depends on the placement of the application's data. The partitioning of a file determines the degree of parallelism in servicing a single request to the file, and the allocation of the file partitions onto disks determines the load balance in the array. Finally, the degree of intra-request parallelism and the load balancing together determine the throughput and the effective speed-up of requests.

\subsection{Outline of the Paper}

The paper presents our approach to automatic tuning of data placement for loosely coupled disk arrays. Section 2 discusses the heuristic methods that we have developed for partitioning files so as to exploit I/O parallelism and for allocating file partitions so as to balance the load across all disks of the array. Section 3 generalizes the load balancing method by considering predictable as well as unpredictable dynamic changes of access characteristics. Finally, Section 4 briefly discusses extensions of our work to include global file reorganizations and application-based data partitioning. 


\section{Automatic Tuning of Data Placement}

The goal of our research on 1/O parallelism is to develop algorithms and to build system software (i.e., a file manager and a low-level storage manager for advanced database systems) that can effectively exploit loosely coupled disks. Since the performance of a disk array depends critically on the placement of data, we have especially investigated algorithms for tuning the placement of files towards the workload characteristics of an application.

In principle, the data placement problem can be viewed as a file assignment problem, which has been intensively studied in many variants and is known to be NP-hard. Hence, viable solutions must be based on a heuristic approach. In the ample work reported on the file assignment problem $[5,25]$ such heuristics have been investigated only for the static case, that is, when all files are to be allocated at the same time and the access characteristics of the files are completely known in advance. Also, the issue of $\mathrm{I} / \mathrm{O}$ parallelism in servicing a single request has been disregarded so far. In advanced data-intensive applications such as object-oriented database systems or multimedia storage servers, data placement methods must take into account the potential performance gains of intra request parallelism as well as its overhead and deal with dynamic file allocations and deletions, with expansions or shrinkage of files, and with unpredictable changes in the access characteristics of existing files. Moreover, since the tuning of data placement should be automated and incorporated into the file manager or database system, practically viable algorithms for file partitioning and allocation must be invoked dynamically at low execution costs.

We have developed such a low-overhead heuristic method for tuning the placement of files on a disk array or conventional multi-disk system [23,24]. This method consists of a file partitioning algorithm based on striping and a file allocation algorithm which aims to ensure good load balancing. The main properties of these two components are outlined in Subsections 2.1 and 2.2 ; the benefits and potential applications of our approach as well as a brief discussion of a performance evaluation are summarized in Subsection 2.3.

\subsection{Data Partitioning based on File Striping}

Our approach to file partitioning is based on striping. In a striped file system, the striping unit must be chosen carefully, as it determines both the degree of intra-request parallelism and the parallelism of independent requests. A large striping unit tends to cluster a file on 


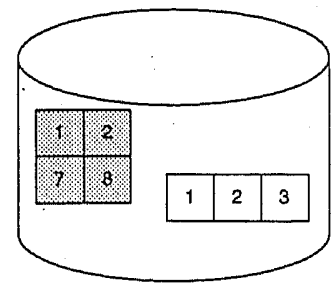

File $_{1}$ (striping unit $=2$ blocks)
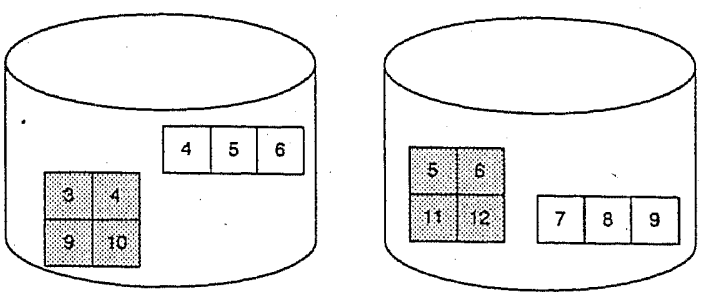

File $_{2}$ (striping unit $=3$ blocks)

Figure 1: File-specific striping units

one disk, so that no intra-request parallelism would be exploited. A small striping unit, on the other hand, increases the total amount of device-busy time that is consumed for servicing a request, because a single request of relatively small size utilizes multiple disk arms [1]. In this case, striping severely limits the maximum achievable throughput of requests.

In virtually all disk array architectures or disk-array-based file systems that have been developed or proposed so far, the striping unit is chosen globally, that is, uniformly for all files. In [2] a heuristic method is proposed to determine the striping unit of a disk array, based on the application's throughput requirements, i.e., knowledge of the average request size and the expected multiprogramming level (in a closed queueing model). This method seems to be suitable for applications such as scientific data repositories or pure on-line transaction processing (OLTP), in which all files have approximately the same sort of access characteristics (i.e., only large requests or only single-block requests). However, it seems that a global striping unit is inappropriate if the access characteristics of files are highly diverse within a single application, for example, in a combined OLTP/batch environment, in an object-oriented or extended relational database system, or in a multimedia storage manager (e.g., for office document filing).

We have designed and built a multi-disk file system prototype, called FIVE ${ }^{1}$ [23], in which the striping unit can be chosen individually for each file or even portions of a file, so that files with different average request sizes can be treated differently. An example of such file-specific striping units is shown in Figure 1. Our system allows relatively small

\footnotetext{
${ }^{1}$ FIVE stands for Flle system with adaptiVe Enhancements.
} 


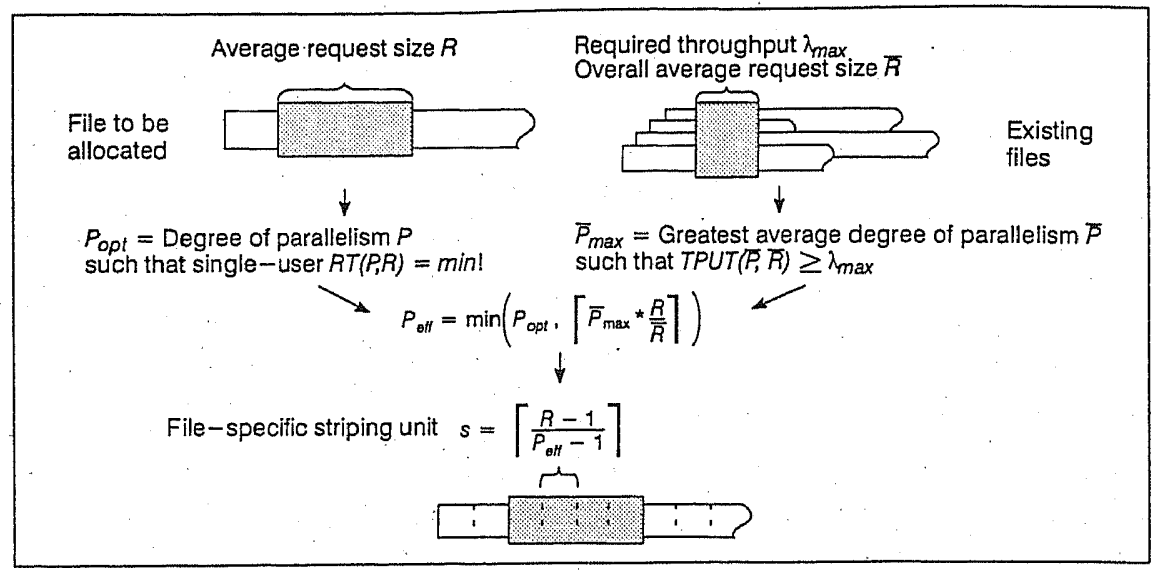

Figure 2: Method to determine the striping unit of a file

striping units for files with very large request sizes and/or critical response time constraints, while using very coarse striping units for other files or even not striping other files at all because of throughput considerations [8]. Moreover, the capability to deal with file-specific striping units is of great advantage if the workload characteristics of the application change over time. In this case, some files (e.g., the most frequently accessed files or the files with the most critical response time) can be individually restriped without having to unload and reload the entire file system.

We have developed a simple analytic model for heuristically determining the optimal striping unit and degree of declustering of a new file, based on an estimate of the file's average request size. The algorithm chooses a degree of parallelism that minimizes the response time of requests subject to the constraint that the achievable throughput of the array is at least as high as the application's arrival rate of requests. The striping unit and the degree of declustering of the file are then derived from this optimal degree of parallelism. This heuristic method for tuning the striping units of files is illustrated in Figure 2. An experimental study [24] indicates that file-specific striping units as determined by our method can significantly improve performance compared to the best possible global striping unit. 


\subsection{Data Allocation and Load Balancing}

Having decided to decluster a file over a particular number of disks, all striping units of the file that would be mapped to the same disk are combined into a single allocation unit called an extent. We then have to select the disks on which the file extents are actually placed. When the various files have different access patterns, an intelligent decision is crucial for disk load balancing. Our system keeps track of a number of related statistics: the heat of extents and disks, where the heat is defined as the sum of the number of block accesses of an extent or disk over a certain period of time, and the temperature of extents, which is defined as the ratio between heat and size.

A simple but effective heuristic algorithm for the static allocation problem, where all files are to be allocated at the same time and the heat of each extent is known or can be estimated in advance, is discussed in [3]. The algorithm first sorts all extents by descending heat, and extents are allocated in this sort order. For each extent allocation, the algorithm selects the disk with the lowest accumulated heat among the disks that have not yet been assigned another extent of the same file.

We have extended this greedy algorithm so as to deal with dynamic allocations. In [23] we have developed a class of disk selection algorithms which provide a good compromise between access time minimization, load balancing and extra $\mathrm{I} / \Theta_{\mathrm{s}}$ due to partial reorganization. Partial disk reorganization may have to be performed if due to file additions and deletions there is room to store a file on a disk, but the space is not contiguous. At one end of our spectrum of disk selection algorithms, we have the option to select the disks in increasing heat order without regard to reorganization costs. At the other end of the spectrum, we may select the disks in multiple passes, where in the first pass we choose only disks which have enough contiguous space. Our algorithms are geared towards a dynamic environment where not only entire files can be added or deleted on request, but also existing files may expand or shrink.

As an example, consider the disk array and the heat distribution shown in Figure 3 , and assume that a new file with a degree of declustering of 8 is to be allocated; the file consists of 8 extents that are to be placed on 8 different disks. If on-line reorganizations are acceptable, then our algorithm would select the disks $d_{1}$ through $d_{8}$, i.e., the 8 coolest disks. This would incur partial disk compactions on disks $d_{1}, d_{2}, d_{4}, d_{5}$, and $d_{7}$; in order to allocate the extents on disks $d_{3}$ and $d_{8}$, sufficient free space would have to be reclaimed on these two disks by 


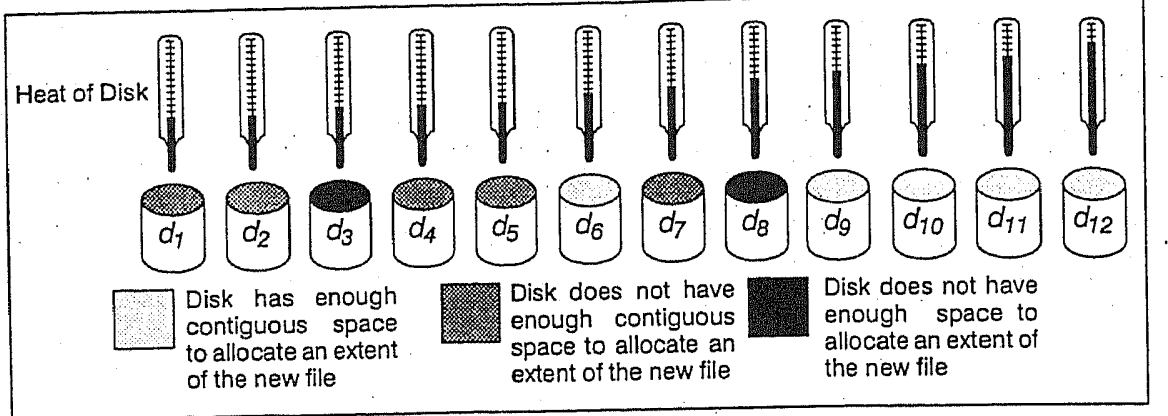

Figure 3: Example of heat and free-space distribution of a disk array

reallocating one or more of their coolest extents onto another disk or by migrating them to tertiary storage (e.g., optical disks or automatic cassette archives). If, on the other hand, on-line reorganizations are to be avoided at any cost, then our algorithm would select the disks $d_{6}, d_{9}, d_{10}, d_{11}, d_{12}, d_{1}, d_{2}, d_{4}$ (in this order). In this case, the load (im)balance in the array would become worse if the newly allocated file turned out to be frequently accessed; however, the allocation of the new file would incur relatively low reorganization costs.

If a certain disk is selected for allocation in a given strategy due to space or access time constraints, our algorithms also allow the invocation of a dynamic load balancing step, called disk cooling. This is basically a greedy algorithm. which removes from the disk a number of extents in decreasing order of temperature and places them on the coolest disks which do not yet hold extents of the corresponding files. The temperature metric is used as the criterion for selecting the extents that are to be reallocated, because temperature reflects. the benefit/cost ratio of a reallocation since benefit is proportional to heat (i.e., reduction of heat) and cost is proportional to size (of the reallocated extents). In our current file system prototype, disk cooling is automatically invoked whenever significant load imbalances are detected in the disk array at allocation or deallocation time.

\subsection{Application and Evaluation of the Method}

The developed heuristic method for file partitioning, file allocation, and dynamic load balancing has the following salient properties: 
- It consists of modular building blocks that can be invoked independently; in particular, the algorithm for file allocation and disk load balancing can be used regardless of whether striping is employed or not.

- It can take into account explicitly specified constraints on data placement for recovery, security and other administrative purposes.

- It uses simple but effective heuristics that incur only little overhead.

- Its constituent algorithms can be invoked on-line, i.e., concurrently with regular requests to existing files.

- It is beneficial for a variety of application classes, including on-line transaction processing (OLTP), relational database systems, object oriented database systems, network file servers, multimedia applications, and scientific applications.

- It is mostly motivated by the evolving disk array technology; however, it is applicable to all sorts of multi-disk architectures.

\subsubsection{Performance evaluation}

The algorithms for file partitioning, file allocation, and dynamic load balancing are implemented in an experimental file system prototype [23]. After a successful performance evaluation based on synthetic workloads $[23,24]$, we performed a comprehensive evaluation, based on I/O traces from the OLTP system of a large Swiss bank (Union Bank of Switzerland). Due to the static nature of this application (new files are allocated only twice a year) and the homogeneous workload with respect to the size of the $1 / 0$ requests (almost all requests were one block in size), the most important issue in this study was that of load balancing.

The on-line database of the bank consists of more than 150 files with a total size of about 23 GBytes. The $1 / 0$ trace recorded more than $550000 \mathrm{I} / 0$ requests to these files. In our performance study, we assumed a priori knowledge of the heat and the average request size of the files. This information, which is relatively stable in the underlying OLTP system, was obtained by pre-analyzing the trace.

We compared three different allocation heuristics using a simulated disk array with 48 disks of capacity 0.5 GBytes each. Space-balancing is a heuristic that selects the disk with the largest available space for allocating a file partition. Round-robin allocates the file partitions 


\begin{tabular}{|l||c|c|c|}
\hline \multicolumn{1}{|c||}{} & \multicolumn{3}{c|}{ Allocation Method } \\
\hline Striping Method & Space-balancing & Round-Robin & Heat-balancing \\
\hline \hline Cylinder (440 KB) & 63 & 61 & 41 \\
\hline Block (8 KB) & 45 & 44 & 31 \\
\hline File-specific & 41 & 42 & 30 \\
\hline No Striping & $(408)$ & $(110)$ & $(70)$ \\
\hline
\end{tabular}

Table 1: Average response time [msec] for different allocation and striping methods

in a circular manner, and thus balances the number of disk partitions per disk. Heat-balancing. selects the disk with the lowest accumulated heat.

We combined these allocation strategies with four different striping methods. The first two striping methods were global ones, i.e., the striping unit was chosen to be the same for all files: one block ( $8 \mathrm{~KB}$ ) or one cylinder ( 55 blocks of $8 \mathrm{~KB}$ each), given that the average request size in our environment was a block. The third method employed file-specific striping units that were chosen using the heuristic method of Section 2.1. Finally, the last method did not use striping at all, i.e., each file was allocated to one disk.

Table 1 shows the average response time of an $1 / 0$ request (in milliseconds) at an average arrival rate of 1100 requests per second. The heat-balancing approach is the clear winner, regardless of the striping method. Heat-balancing outperformed the other two allocation methods by more than $30 \%$. This shows that striping alone (i.e., combined with a very simple allocation heuristic like Round-robin) does not guarantee good load balancing.

Without striping, however, we observed a disastrous response time degradation. In the case with no striping, we had to reduce the arrival rate of the requests by a factor of 5 ; with the original arrival rate, the system was not able to handle the load. In this case, the disks with the hottest files became a premature bottleneck and limited the performance of the disk array. So file partitioning seems to be necessary to obtain a sufficient number of allocation. units to be distributed by the heat-balancing heuristic.

With a striping unit of one block, the system performed slightly better compared to a striping unit of one cylinder. This effect was due to the fact that the distribution of block access frequencies was heavily skewed within files. Block striping did a better job on distributing the hot partitions of files across multiple disks, and thus achieved much better load balancing than the cylinder striping method. The file-specific striping method. 


\begin{tabular}{|l||c|c|c|c|}
\hline \multicolumn{1}{|c||}{} & \multicolumn{4}{c|}{ Disk utilization } \\
\hline Allocation Method & Min & Avg & Max & Standard deviation \\
\hline \hline Space-balancing & 0.448 & 0.502 & 0.626 & 0.04 \\
\hline Round-robin & 0.432 & 0.501 & 0.612 & 0.03 \\
\hline Heat-balancing & 0.431 & 0.441 & 0.448 & 0.004 \\
\hline
\end{tabular}

Table 2: Disk utilization for different allocation methods

performed only slightly better than a global striping unit of one block, since most requests were single-block requests. However, for particular files with few but large requests, filespecific striping achieved significant performance gains.

The load balancing achieved by the various allocation heuristics is directly reflected in the disk utilization of the array, as summarized in Table 2 (with a striping unit of one block).

\section{Generalized Load Balancing}

Our original framework[23] allowed for the various files to have different access patterns, but the access pattern of a given file was considered fixed in time. In practice, however, this is not the case, rather we encounter many environments in which the heat of the files changes over time, and, further, some files exhibit a periodical pattern of heat changes. Under these conditions, load balancing needs to be performed even if no striping is used, i.e., the files are allocated in their entirety to a single disk. Thus, it becomes necessary to invoke a generalized disk cooling procedure periodically, not only at file allocation time. We also need to consider explicitly the cost of performing the cooling, which was not included in our earlier model, as well as use a generalized objective function which represents the variance of the disk array heat over a certain interval of time.

We distinguish between Predictable Heat Files (PHF), which exhibit a periodical heat pattern over a given cycle, and Unpredictable Heat Files (UHF), for which we can observe only the current heat. The optimization time span, TS, of the load balancing procedure can be chosen, as shown in Figure 4, as the maximum cycle of all the cycles of the PHFs. The union of the points in time where the individual PHFs change heat patterns induces a partition of TS into $n$ time intervals. Thus, mare formally we can define TS as the vector: TS $=\left[\left(t_{1}-t_{0}\right),\left(t_{2}-t_{1}\right), \ldots\left(t_{n}-t_{n-1}\right)\right]$ and denote by interval $j$ the interval of length $\left(t_{j}-t_{j-1}\right)$. 
Let us also denote by $H(i, j)$ the heat of disk $i$ in interval $j$. Correspondingly, the weighted disk heat of disk $i$ over the time span TS is defined as:

$$
W D H(i, T S)=\sum_{j=1}^{n} H(i, j) *\left(t_{j}-t_{j-1}\right) .
$$

The objective cost function to be minimized by our generalized load balancing procedure is the Weighted Disk Heat Variance (WDHV) over the time span TS:

$$
W D H V(H, T S)=\sum_{j=1}^{n} \sum_{i=1}^{D}(\vec{H}(j)-H(i, j))^{2} *\left(t_{j}-t_{j-1}\right)
$$

where $H$ is a matrix whose element in position $(i, j)$ is $H(i, j)$

$$
\begin{aligned}
& D=\text { number of disks in the array } \\
& \vec{H}(\mathrm{j})=\sum_{i=1}^{D} \mathrm{H}(\mathrm{i}, \mathrm{j}) / \mathrm{D}
\end{aligned}
$$

We will consider a number of heuristic strategies for generalized disk cooling. In a serial strategy, we will determine a sequence of beneficial actions to be executed immediately. Let us define an action as a record type with the following components:

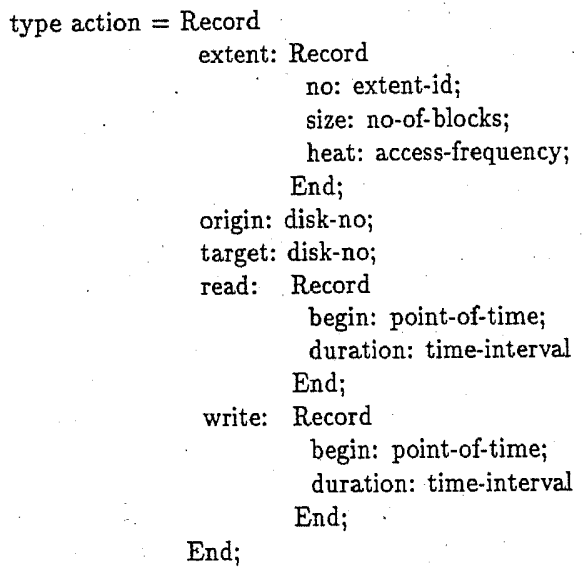

Our algorithm is basically a greedy procedure that for each consecutive action determines the extent to be removed (action.extent), by picking the disk with the highest weighted heat (action.origin) and then choosing the extent with the highest weighted temperature. We then determine to which disk the extent should be moved (action.target), by choosing the disk with the lowest weighted heat subject to some constraint. Let us denote by $W D H V^{\prime}$ the weighted disk heat variance over the time span TS after a given action is executed: 


$$
W D H V^{\prime}(H, T, \text { action })=\sum_{j=1}^{n} \sum_{i=1}^{D}\left(\bar{H}^{*}(j)-H^{*}(i, j)\right)^{2} *\left(t_{j}-t_{j-1}\right)
$$

where $H^{*}(i, j)=H(i, j)$ if $\mathrm{i} \neq$ action.target or action.origin

$$
\begin{aligned}
& H^{*}(i, j)=H(i, j)+\text { action.extent.heat if } \mathrm{i}=\text { action.target } \\
& H^{*}(i, j)=H(i, j)-\text { action.extent.heat if } \mathrm{i}=\text { action.origin }
\end{aligned}
$$

A high level version of the Generalized Disk Cooling algorithm (GDC) is given in Figure 5. In order to determine whether a given action is beneficial or not we have to consider not only the reduction in heat on the origin disk and the addition in heat on the target disk, but also the additional heat caused by the reorganization process itself as shown in Figure 6 a. The cooling process is executed during two intervals of time, the first corresponding to the read phase of the action and the second corresponding to the write phase of the action. The read and write phases introduce an amount of additional of heat on the origin and target

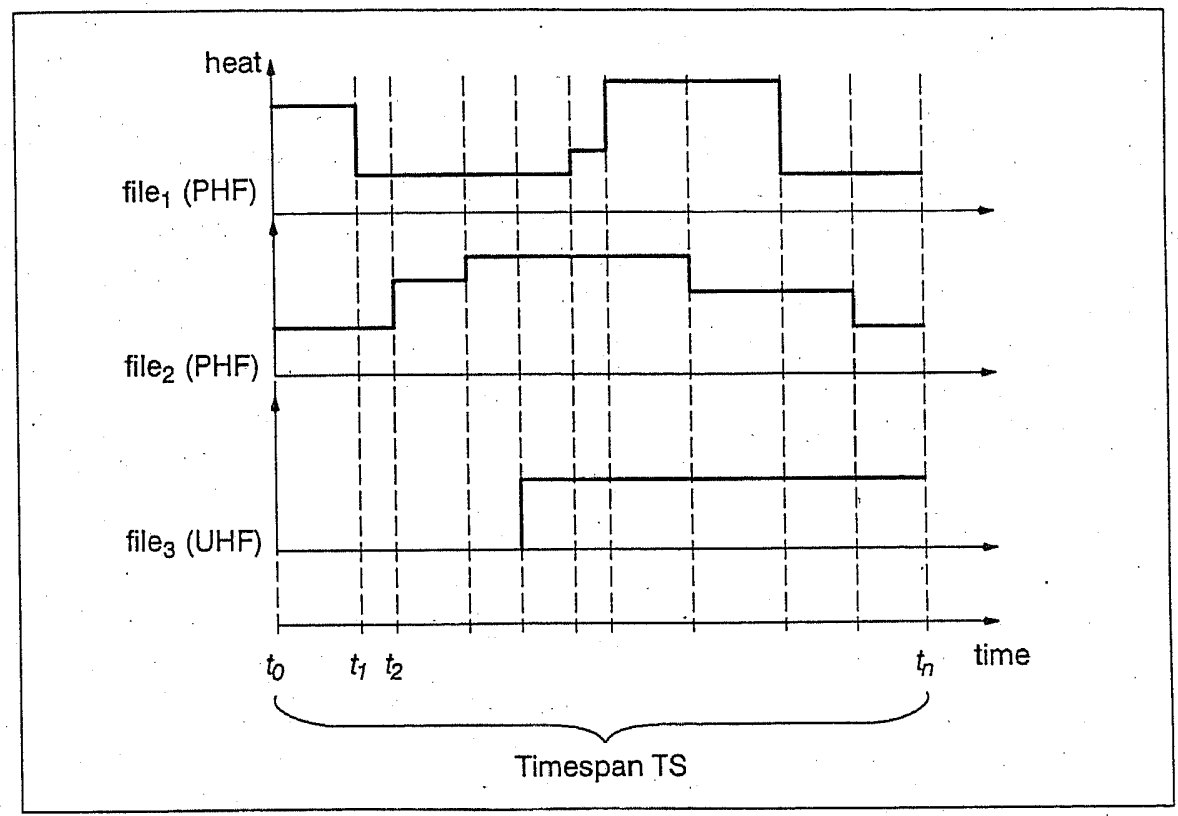

Figure 4: Temporal heat changes of predictable-heat and unpredictable-heat files 
Generalized Disk Cooling Algorithm (GDC)

Input: $D$ - number of disks

Matrix $H-H(i, j)$ is heat of disk $D_{i}$ in interval $j$;

Matrix $T-T(k, j)$ is the temperature of extent $e_{k}$ in interval $j$

Vector $T S$ - intervals of time span;

$t_{\text {cur }}$

- current point in life cycle;

Output: actions-to-do-list of cooling actions;

benefit $=$ true;

actions-to-do = empty;

start-next-action $=t_{\text {cur }}$;

While benefit $=$ true do

Step 0: If trigger-condition = true then execute remaining steps;

Step 1: Choose new extent and origin disk:

action.origin $=$ the disk with the highest weighted. heat;

action.extent.no $=$ the extent on action.origin with the highest weighted temperature;

Step 2: Choose new target disk:

action.target $=$ the disk with the lowest weighted heat such that this disk does not hold already an extent of the file to which action.extent belongs;

Step 3: Estimate the cooling time:

action.read.begin $=$ the point in time at which the read phase of the cooling action will start $=$ start-next-action;

action.read.duration $=$ the time it takes to read action.extent from action.origin into memory

action.write.begin $=$ start-next-action + action.read.duration

action.write.duration $=$ the time it takes to write action.extent onto action.target

Step 4: Determine the intervals $m$ and $n$ when action.read.begin

and action.write.begin occur;

Step 5: Calculate $E$, the Extra-Weighted Disk Heat Variance due to action:

$E=\sum_{i=1}^{D}\left(\bar{H}^{\prime}(m)-H^{\prime}(i, m)\right)^{2} *$ action.read.duration

$\sum_{i=1}^{D}\left(\bar{H}^{\prime \prime}(n)-H^{\prime \prime}(i, n)\right)^{2} *$ action.write.duration;

Step 6: Compute $W D H V$, the current weighted disk heat variance, and $W D H V^{\prime}$, the weighted disk heat variance if action is to be executed ;

Step 7: If $W D H V-\left(W D H V^{\prime}+E\right)>$ Epsilon

then

add action to actions-to-do

start-next-action $=$ start-next-action +

action.read.duration + action.write.duration

endwhile

else benefit $=$ false;

Figure 5: GDC algorithm 
disks which is computed by dividing the size of the extent to be moved by the corresponding duration of the phase.

The duration times of the read and write phases of an action can be calculated by making use of an $M / G / 1$ queueing model with a non-preemptive prioritized server. We consider that our system consists of requests with two priorities: regular user requests have high priority, while the cooling requests have low priority. Cooling requests represent the read/write phases of the cooling actions. Let $L_{c}$ denote the proportion of disk utilization due to cooling requests and $L_{r}$ the proportion of disk utilization due to regular requests. Then, $R T_{c}$, the response time of a cooling request with a non-preemptive server is given by [9]:

$$
R T_{c}=\frac{k L T}{(1-L)\left(1-L_{r}\right)}+T_{c}
$$

where $L$ is the total disk utilization $\left(L=L_{c}+L_{r}\right), T$ is the average service time for requests of both priorities, $T_{c}$ is the average service time for cooling requests and $k$ denotes the time to finish the current request when a new one arrives. The distribution coefficient of the service time, $k$, is given by the Khintchine and Pollaczek equation as $k=\frac{1}{2} * \frac{E\left(T^{2}\right)}{T^{2}}$.

In an open queueing model, $L_{r}$, the disk utilization due to regular requests, can be calculated as: $L_{r}=T_{r} * \lambda_{r}$, where $T_{r}$ is the average service time for regular requests and $\lambda_{r}$ is the corresponding arrival rate. If we assume that the cooling request is currently performed on disk $i$ and that it occurs during time interval $m$, then $\lambda_{r}$ can be computed by using the formula:

$$
\lambda_{r}=\frac{H(i, m)}{\bar{R}} \text { where } \bar{R} \text { is the average request size. }
$$

Since cooling requests do not have an observable arrival rate, we shall estimate $\lambda_{c}$ by the following upper bound:

$$
\lambda_{c} \leq \frac{1}{\text { (optimal service time for avg cooling request) }}
$$

In step 4 of the GDC algorithm we compute $E$, the Extra-Weighted Disk Heat Variance, by making use of the following approximation technique. In order to avoid splitting the current time interval, and possibly the next one (see Figure 6a), and thus incurring expensive recalculations of $W D H V$, we add two "dummy" intervals to the load balancing cycle to account for the two cooling phases of the action, as shown in Figure 6b. During such a dummy interval the heat of each disk, except for the disk which is the subject of the cooling operation, is taken to be the same as the heat of the disk during the time interval when 


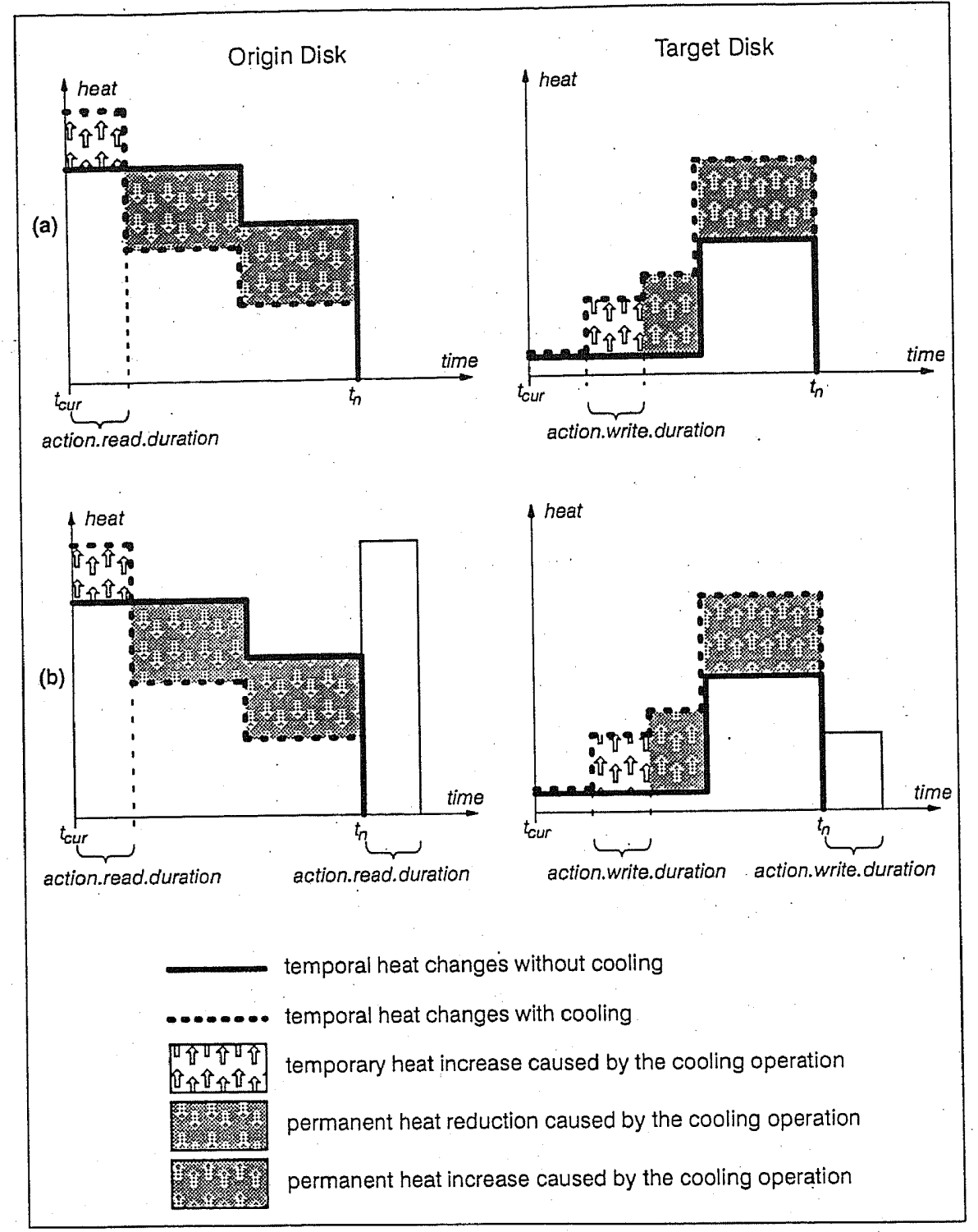

Figure 6: (a) Cost and benefit of cooling, (b) adjustment of weighted heat variance 
the corresponding cooling process started. Thus, the extra contributions to the WDHV are computed as given below:

$H^{\prime}(i, j)=H(i, j)$ +action.extent.size/action.read.duration for $\mathrm{i}=$ action.origin $H^{\prime}(i, j)=H(i, j)$ otherwise $H^{\prime \prime}(i, j)=H(i, j)+$ action.extent.size/action.write.duration for $\mathrm{i}=$ action.target $H^{\prime \prime}(i, j)=H(i, j)$ otherwise.

We are currently implementing the GDC algorithm in our FIVE system [23] and we will perform comprehensive experiments with various synthetic and real workloads. We plan to investigate when to trigger this cooling procedure and whether the time span TS for the optimization should be equal to the maximum life cycle of the PHFs or shorter. We also will develop and experiment with a parallel disk r voling algorithm where extents can be removed or allocated in parallel, subject to certain constraints with regard to the amount of buffer space available in memory.

\section{Further Extensions of Our Method}

\subsection{Global File Reorganization and Restriping}

Due to dynamic growth and shrinkage, some files may end up having multiple extents on the same disk. A global file reorganization procedure could then be invoked in order to improve the seek time by reallocating the multiple extents of the file(s) that reside on the same disk such as to achieve physical proximity, similar to the procedure used for record clustering in [18]. However, a more cost effective reason for a reorganization may be dictated by restriping needs. If it is determined that the average request size of a file changes over time, then the file should be reallocated with a different striping unit and/or different degree of declustering in order to improve the response time or to meet the application's throughput requirements. Since our file allocation strategy assigns different striping units and degrees of declustering to individual files, the restriping can be done on a file basis, thus allowing the reorganization process to run concurrently with user transactions [17]. When dealing with large disks, the reorganization often is performed in place due to space restrictions [22]. As opposed to this approach, we are investigating a restriping algorithm that always attempts to choose new disks for the reallocation in order to achieve the maximum degree of parallelism between the 
read and write phases of the reorganization and to maintain a good load balancing. We plan to study how the original file allocation algorithm proposed in [23] can be incorporated in the restriping procedure in order to account for the various free-space states of a disk.

\subsection{Application-based Declustering}

So far, our work has concentrated on partitioning at the block level. This is generally a feasible approach since all data types are ultimately mapped into block-strụctured files. However, further performance gains may be obtained by employing partitioning schemes that are directly based on the application's data types. For example, in a relational database system, tables can be partitioned into fragments based on attribute values by applying a hash function or by range-partitioning the domain of the corresponding attribute $[4,6,14]$. In applications that deal with complex objects (e.g., CAD or office information systems), a variety of partitioning schemes need to be considered based on the various access patterns. In addition, in some of these applications the partitioning is subject to real-time constraints dictated by visualization requirements (e.g, multimedia applications, scientific data repositories).

We plan to investigate such application-based partitioning schemes and to develop heuristics for optimal partitioning of complex objects and sets of complex objects. Furthermore, we intend to develop appropriate mapping strategies from application-based partitioning schemes to our block-level partitioning scheme. We note here that our method for data allocation and disk load balancing consists of flexible generic procedures that can be used with any application-based partitioning scheme. 


\section{References}

[1] Bitton, D. and Gray,J.N., "Disk shadowing," Proc. 14th International Conference on Very Large Databases, 1988, pp. $331-338$.

[2] Chen, P.M., and Patterson, D.A., "Maximizing Performance in a Striped Disk-Array," Proceedings of the 17th International Symposium on Computer Architecture; 1990, pp. $322-331$.

[3] Copeland, G., Alexander, W., Boughter E., and Keller, T., "Data Placement in Bubba," Proc. ACM International Conference on Management of Data, 1988, pp. 99- 108.

[4] DeWitt,D.J., and Gray, J.N., "Parallel Database Systems," ACM SIGMOD Record, Vol. 19, No. 4, 1990.

[5] Dowdy, L.W., and Foster, D.V., "Comparative Models of the File Assignment Problem," ACM Computing Surveys, Vol. 14, No. 2, June 1982, pp. 95 - 121.

[6] Ghandeharizadeh, S., and DeWitt, D.J., "Hybrid-Range Partitioning Strategy: A New Declustering Strategy for Multiprocessor Database Machines," Proc. 16th International Conference on Very Large Data Bases, 1990, pp. 481 - 492.

[7] Gibson, G.A., Hellerstein, L., Karp, R.M., Katz, R.H. and Patterson, D.A., "Failure Correction Techniques for Large Disk Arrays", Proc. Third ACM Intern. Conf., on Architectural Support for Programming Languages and Operating Systems, 1989, pp. $123-132$.

[8] Gray, J.N., Horst B., and Walker, M., "Parity Striping of Disc Arrays: Low-Cost Reliable Storage with Acceptable Throughput," Proc. 16th International Conference on Very Large Data Bases, 1990, pp. 148 - 161.

[9] Highleyman, W., Performance Analysis of Transaction Processing Systems, Prentice Hall, 1989.

[10] Hsiao, H. and DeWitt, D.J., "Chained Declustering: A New Availability Strategy for Multiprocessor Database Machines," Proc. Sixth IEEE International Conference on Data Engineering, 1990, pp. $456-465$. 
[11] Katz, R.H.," High Performance Network and Channel-based Storage," Sequoia 2000 Technical Report \#91/2, University of California at Berkeley, 1991.

[12] Katz, R.H., Gibson, G.A., and Patterson, D.A., "Disk System Architectures for High Performance Computing," Proc. of the IEEE, Vol. 77, No. 12, 1989, pp. $1842-1858$.

[13] Kim, M.Y., "Synchronized Disk Interleaving," IEEE Transactions on Computers, Vol. C-35, No. 11, 1986, pp. $978-988$.

[14] Livny, M., Khoshafian, S., and Boral, H., "Multi-Disk Management Algorithms," ACM SIGMETRICS Conference, 1987, pp. 69 - 77.

[15] Moad, J., "Relief for Slow Storage Systems," Datamation, September 1, 1990.

[16] Muntz, R.R. and Lui, J.C., "Performance Analysis of Disk Arrays Under Failure," Proc. 16th International Conference on Very large Databases, 1990, pp. $162-173$.

[17] Omiecinski, E., Lei, L. and Scheuermann, P., "Concurrent File Reorganization for Record Clustering: A Performance Study," Proc. Eight International Conference on Data Engineering, 1992, pp. 265 - 272.

[18] Omiecinski, E. and Scheuermann, P., "A Parallel Algorithm for Record Clustering," ACM Transactions on Database Systems, Vol. 15, No, 9, December 1990, pp. 569 624.

[19] Patterson, D.A., Gibson, G.; and Katz, R.H., "A Case for Redundant Arrays of Inexpensive Disks (RAID)," Proc. ACM SIGMOD International Conference on Management of Data, 1988, pp. $109-116$.

[20] Salem, K., and Garcia-Molina, H., "Disk Striping," Proc. Second International Conference on Data Engineering, 1986, pp. $336-342$.

[21] Sierra, H.M., An Introduction to Direct Access Storage Devices, Academic Press, San Diego, CA, 1990

[22] Scheuermann, P., Park, Y.C. and Omiecinski, E., "A Heuristic File Reorganization Algorithm based on Record Clustering," BIT, Vol.29, 1989, pp. $428-437$. 
[23] Weikum, G., Zabback, P., and Scheuermann, P., "Dynamic File Allocation in Disk Arrays," Proc. ACM SIGMOD International Conference on Management of Data, 1991, pp. $406-415$ (extended version available as: Technical Report No. 147, Computer Science Dept., ETH Zurich, 1990).

[24] Weikum, G., and Zabback, P., "Tuning of Striping Units in Disk-Array-Based File Systems," Proc. Second International Workshop on Research Issues on Data Engineering: Transaction and Query Processing (RIDE-TQP), 1992, pp. $80-87$

[25] Wolf, J., "The Placement Optimization Program: A Practical Solution to the Disk File Assignment Problem," Proc. ACM SIGMETRICS and Performance'89 International Conference on Measurement and Modeling of Computer Systems, Berkeley, 1989, pp. $1-10$. 
Gelbe Berichte des Departements Informatik

157 T. Weibel, G. Gonnet

158 M. Scholl (ed.)

$159 \quad$ K. Gates

160

H. Mössenböck

161 S. Lalis

162

163

164 G.H. Gonnet, H. Straub

165

166

167 H.E. Meier

B. Heeb, I. Noack

169 M. Bronstein

170 P. Arbenz

171 K. Simon, P. Trunz

172 A. Rosenthal, Ch. Rich, M.H. Scholl

173 P. Schäuble, B. Wüthrich

174 M. Brandis, R. Crelier

M. Franz, J. Templ
An Algebra of Properties

Grundlagen von Datenbanken (Kurfassungen

des 3.Gi-Workshops, Volkse, 21. - 24.5.91)

Using Inverse Iteration to Improve the Divide and Conquer Algorithm

Differences between Oberon and Oberon-2

The programming Language Oberon-2 (vergriffen)

XNet: Supporting Distributed Programming in the Oberon Environment (vergriffen)

Multi-Level Transaction Management for Complex Objects: Implementation, Performance, Parallelism

eXperimental geometrY Zurich: Software for Geometric Computation

The Dynamic Programming Algorithm as a Finite Automation

Towards a Divide and Conquer Algorithm for the Real Nonsymmetric.Eigenvalue Problem

N-Poisson Document Modelling Revisited

Schriftgestaltung mit Hilfe des Computers

Typographische Grundregeln mit Gestaltungsbeispielen (neue erweiterte Auflage)

Hardware Description of the Workstation Ceres-3

Formulas for Series Computations

Divide and Conquer Algorithms for the Bandsymmetric Eigenvalue Problem

On Transitive Orientation

Reducing Duplicate Work in Relational Join(s): A Unified Approach

On the Expressive Power of Query Languages

The Oberon System Family 